\title{
Available Phosphorus Levels in Diets for Muscovy Ducks in Housing*
}

http://dx.doi.org/10.1590/1806-9061-2018-0914

\section{-Author(s)}

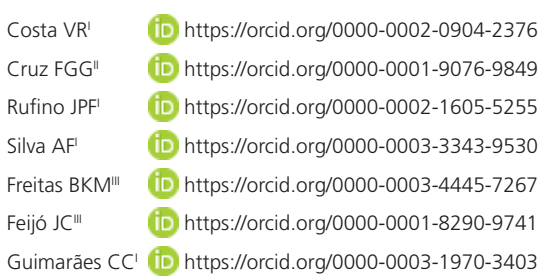

Graduate Program in Animal Science, College of Agrarian Sciences, Federal University of Amazonas, Manaus, Amazonas, Brazil.

" Department of Animal and Vegetable Production, College of Agrarian Sciences, Federal University of Amazonas, Manaus, Amazonas, Brazil.

III Animal Science undergraduate, College of Agrarian Sciences, Federal University of Amazonas, Manaus, Amazonas, Brazil.

*Part of Master's degree dissertation of the first author.

\section{-Mail Address}

Corresponding author e-mail address Frank George Guimarães Cruz

Department of Animal and Plant Production, College of Agrarian Sciences, Federal University of Amazonas, South Sector, Universitary Campus, Av. General Rodrigo Octávio Jordão Ramos, 6200, Coroado I, Manaus, AM, Brazil, CEP 69077-000.

Phone: + 55 xx 92 3305-1181 R. 4082

Email: frankgcruz@gmail.com

\section{- Keywords}

Bone, Cairinamoschata domesticus, carcass, mineral, waterfowl.

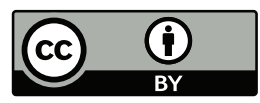

Submitted: 01/December/2018 Approved: 08/March/2019

\section{ABSTRACT}

The present study aimed to determine ideal levels of available phosphorus for muscovy ducks in housing. Two hundred and forty muscovy ducks of creole lineage were used, distributed in boxes with water and food ad libitum. The experimental design was completely randomized with treatments consisting of six nutritional plans that included the initial, growth and termination phases and differed in relation to available phosphorus levels, and four replicates of 10 muscovy ducks each. The birds had weekly performance evaluations, and after 90 days, eight birds (four males and four females) in each treatment were slaughtered for evaluation of carcass traits. Data collected were subjected to Tukey test at $5 \%$ of significance. Differences were not observed ( $p>0.05)$ in performance. Higher available phosphorus levels presented a positive influence $(p<0.05)$ on carcass. Results presented differences $(p<0.05)$ among sexes for carcass development and commercial cuts, with better feed efficiency of males than females in same period. For mineral composition, differences $(p<0.05)$ were observed to calcium (\%), phosphorus (\%) and Ca:Pratio. The present study indicates that nutritional plan 2 (initial $=0.60 \%$; growth $=0.55 \%$ and termination $=0.50 \%$ ) presented ideal nutritional requirement of available phosphorus for muscovy ducks in housing, with better carcass development and mineral deposition on bones.

\section{INTRODUCTION}

Muscovy ducks have the peculiar feature to provide for poultry industry a range of products as meat, eggs, feathers for ornamental purposes, fatty livers and many other products (Rufino et al., 2017). These represent a great market opportunity, but little explored in Latin America (Industrial Poultry, 2005).

There are not many companies that produce muscovy ducks in Brazil, especially due the lack of information's about adequate nutritional requirements, facilities and ideal management (Santos et al., 2012). Brazilian south region concentrates all national production of ducks, muscovy ducks and their derivatives. Only a little piece of this production is destined for internal consumption (ABPA, 2018).

Santa Catarina State is the largest Brazilian producer and exporter of Muscovy duck meat. This meat is especially consumed by the United States, Japan, Angola, Liberia and countries with Arabic ethnicity (Wawro et al., 2004; ABPA, 2018).

According to Mariante et al. (2011) and Gois etal. (2012), the muscovy ducks are waterfowl with great rusticity, presenting exceptionally resistance to diseases and adverse conditions. Physiologically, like other birds, the muscovy ducks require small amounts of minerals, especially phosphorus, that is the second most abundant mineral in its 
tissue composition, with $80 \%$ present in the bones, presenting vital functions to the organism (Pinheiro et al., 2011).

Dunbar et al. (2005) affirms that calcium and phosphorus are independent minerals, and the lack or excess of one can damage the absorption or use of the other, damaging the better performance of birds. And according to Pinheiro et al. (2011), studies that report ideal requirements of available phosphorus for muscovy ducks in literature are very scarce, being used requirements of broilers for its.

Considering the above, the present study aimed to determine ideal levels of available phosphorus for muscovy ducks in housing.

\section{MATERIAL AND METHODS}

This study was conducted in the facilities of the Poultry Sector, Department of Animal and Vegetable Production (DPAV), College of Agrarian Sciences (FCA), Federal University of Amazonas (UFAM), south sector of the University Campus, Manaus/AM, Brazil. The experimental procedures were approved by the Committee for Ethical Animal Use (CEUA - protocol number 017/2016) of Federal University of Amazonas.

Two hundred and forty muscovy ducks (Cairinamoschata domesticus) of creole lineage were used distributed in boxes with water and food ad libitum. The experimental design was completely randomized with treatments consisting of six nutritional plans that included the initial, growth and termination phases and differed in relation to available phosphorus levels (Table 1), and four replicates of 10 muscovy ducks each.

Table 1 - Experimental levels of available phosphorus.

\begin{tabular}{lccc}
\hline Treatments & \multicolumn{3}{c}{ Levels of Available Phosphorus (\%) } \\
\cline { 2 - 4 } & $\begin{array}{c}\text { Initial } \\
(1-35 \text { days })\end{array}$ & $\begin{array}{c}\text { Growth } \\
(36-70 \text { days })\end{array}$ & $\begin{array}{c}\text { Termination } \\
(71-90 \text { days })\end{array}$ \\
\hline Nut. Plan 1 & 0.65 & 0.60 & 0.55 \\
Nut. Plan 2 & 0.60 & 0.55 & 0.50 \\
Nut. Plan 3 & 0.55 & 0.50 & 0.45 \\
Nut. Plan 4 & 0.50 & 0.45 & 0.40 \\
Nut. Plan 5 & 0.45 & 0.40 & 0.35 \\
Nut. Plan 6 & 0.40 & 0.35 & 0.30 \\
\hline
\end{tabular}

Experimental diets (Table 2) were calculated according to the reference values provided by Rostagno et al. (2011), except energy and protein (Rufino et al., 2015) and calcium (Feijó et al., 2016) that used appropriate requirements for muscovy ducks.

Birds started the experimental period with one day of age and were evaluated at 90 days. For performance, the feed intake ( $\mathrm{kg} / \mathrm{bird})$, weight gain ( $\mathrm{kg} / \mathrm{bird}$ ) and feed conversion $(\mathrm{kg} / \mathrm{kg}$ ) were analysed. Due to extreme difficulty of performing the sexing of muscovy ducks with one day, and the lack of a technique for this (Rufino et al., 2017), performance was measured in mixed lots (birds with both sex in the same box).

At 90 days of age, already with an evident sexual dimorphism, after 12 hours of fasting, eight birds of each treatment (four males and four females) were randomly selected, identified and weighed. Next, these were electrically stunned $(40 \mathrm{~V} ; 50 \mathrm{~Hz})$ and slaughtered by cut of jugular vein. The carcasses were immersed into hot water $\left(60^{\circ} \mathrm{C}\right.$ for $\left.62 \mathrm{~s}\right)$, plucked and eviscerated according Mendes \& Patricio's (2004) recommendations, and the carcass yield was determined. Edible viscera (heart, gizzard, pro-ventricle and liver) were individually weighed.

Breast and leg (thigh + drumstick) samples were collected to measure $\mathrm{pH}$ and physical measurements (length, height and width). The commercial cuts (neck, breast, wing, back, thigh and drumstick) were separated according Gomide et al. (2012) and measured by weighing in analytical balance $0.01 \mathrm{~g}$.

Four tibia samples of males per treatment were collected for bone resistance analysis. These were evaluated in Materials Engineering Laboratory of the State University of Amazonas. The bones were cleaned and analysed in a Universal Machine of Electronic Mechanics (Instron Model 5984, with load capacity of $150 \mathrm{KN}$ ) and the data registered for a computer software with results expressed in $\mathrm{kgf} / \mathrm{mm}$ and $\mathrm{N}$. The load applied was 2000 Newton in the central region of bones and the descent rate of the load was $5 \mathrm{~mm} / \mathrm{s}$, with the force applied at the moment before the bone rupture was recorded.

After, the bone's mineral composition (ashes (\%), calcium (\%) and phosphorus (\%)) was evaluated in the EMBRAPA Western Amazon according the methodology proposed by AOAC (1999).

Statistical analysis was performed using the software Statistical Analysis System (2008) and estimates of the treatments were subjected to Tukey test at $5 \%$ of significance.

\section{RESULTS}

Results of performance are present in Table 3. Differences were not observed $(p>0.05)$ in all variables analyzed. Most higher levels of available phosphorus in diets presented better results of feed intake and feed conversion. 


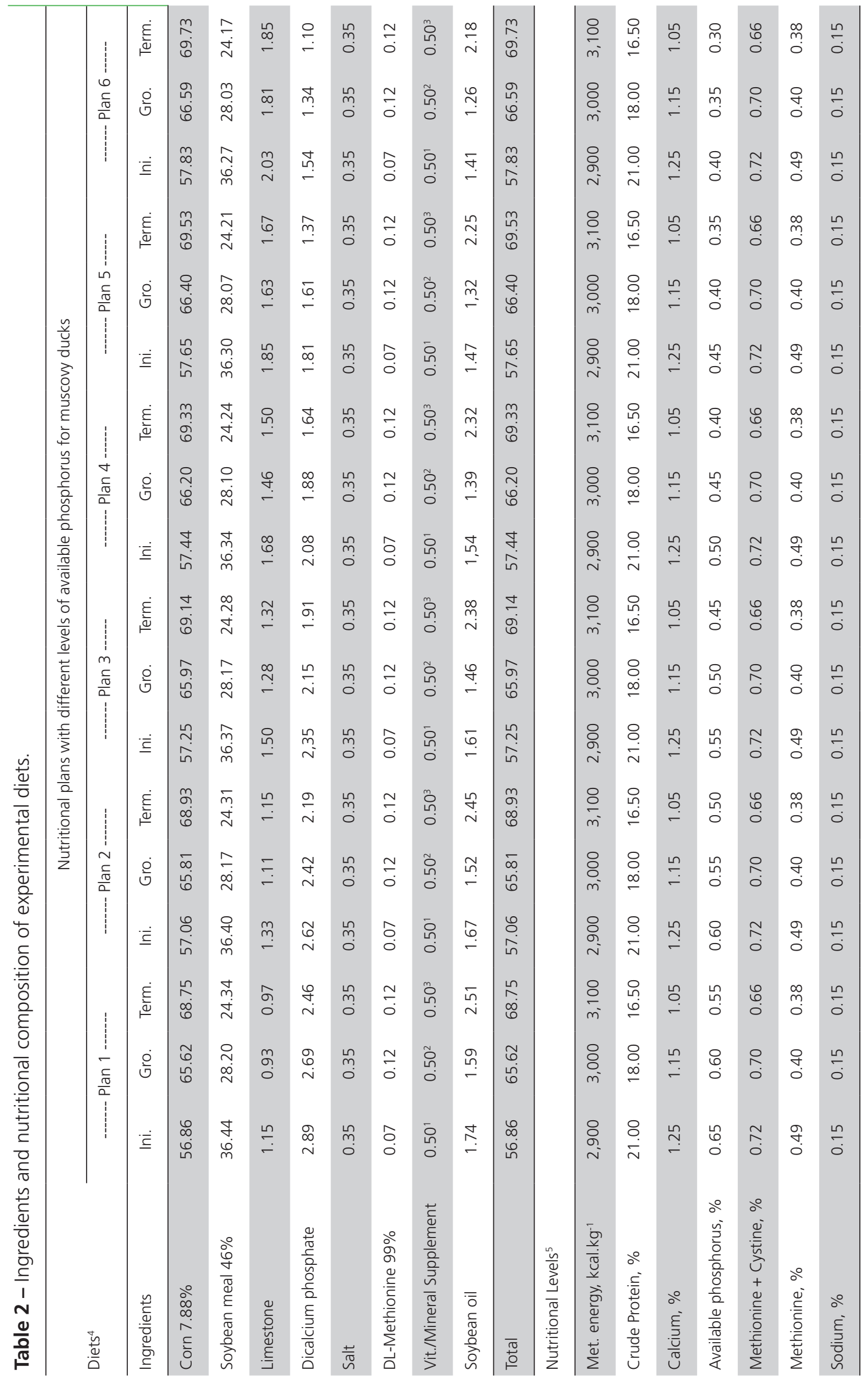

ثं कृष्

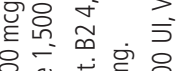

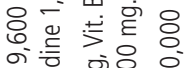

근 홍유

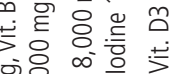

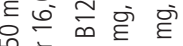

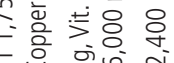

m

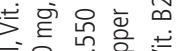

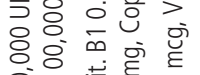

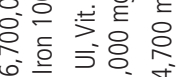

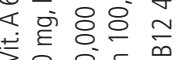

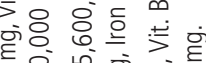

인

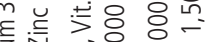

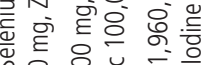

ơ

잉

峁岕

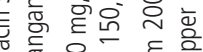

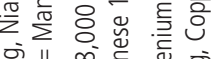

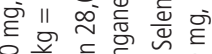

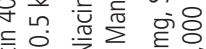

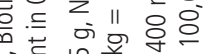

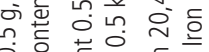

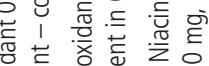

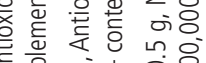

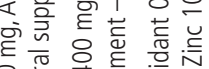

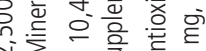

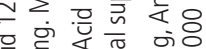

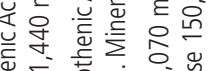

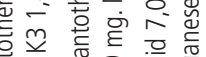

눈

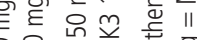

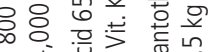

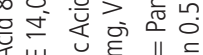

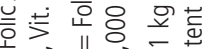

포 응.

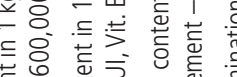

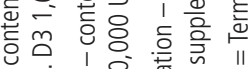

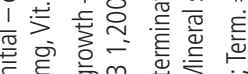

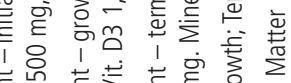

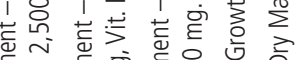

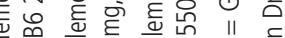

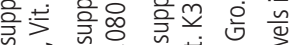

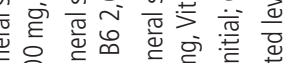

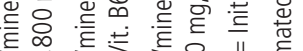

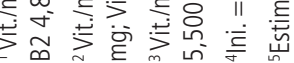


Table 3 - Performance of muscovy ducks in housing fed nutritional plans with different levels of available phosphorus.

\begin{tabular}{lccc}
\hline Nutritional Plans & \multicolumn{3}{c}{ Variables } \\
\cline { 2 - 4 } & $\begin{array}{c}\text { Feed intake } \\
(\mathrm{g})\end{array}$ & $\begin{array}{c}\text { Weight gain } \\
(\mathrm{g})\end{array}$ & $\begin{array}{c}\text { Feed conversion } \\
(\mathrm{kg} / \mathrm{kg})\end{array}$ \\
\hline Nut. Plan 1 & $8,596.50$ & $2,357.49$ & 3.64 \\
Nut. Plan 2 & $8,043.93$ & $2,309.49$ & 3.48 \\
\hline Nut. Plan 3 & $8,396.25$ & $2,340.63$ & 3.58 \\
\hline Nut. Plan 4 & $8,538.25$ & $2,409.96$ & 3.55 \\
\hline Nut. Plan 5 & $9,110.91$ & $2,529.52$ & 3.61 \\
\hline Nut. Plan 6 & $8,891.83$ & $2,447.87$ & 3.63 \\
\hline$p$-value & $0.58^{\text {ns }}$ & $0.72^{\text {ns }}$ & $0.86^{\text {ns }}$ \\
\hline CV (\%) & 9.95 & 8.93 & 9.04 \\
\hline
\end{tabular}

$\mathrm{CV}=$ Coefficient of variation; $\mathrm{ns}=$ non-significant.
Results of carcass traits are present in table 4. Differences $(p<0.05)$ were observed for slaughter weight, foot and gizzard among nutritional plans, and for all variables among sexes.

Birds fed nutritional plans 1 and 3 presented better carcass results. Birds fed nutritional plans with lower levels of available phosphorus presented worse development of carcass. Male muscovy ducks presented better development of carcass, with great difference in the development of carcass among sexes. There was not interaction ( $p>0.05$ ) between factors.

Results of commercial cuts are present in table 5 . Differences $(p<0.05)$ were observed for the $\%$ of thigh among nutritional plans, and for all variables among sexes.

Table 4 - Slaughter weight (SW), carcass yield (CY), feathers (FE), foot (FT), abdominal fat (AF), liver (LV), heart (HT), gizzard (GZ) and pro-ventricle (PV)of muscovy ducks in housing fed nutritional plans with different levels of available phosphorus.

\begin{tabular}{|c|c|c|c|c|c|c|c|c|c|}
\hline \multirow[b]{2}{*}{ Factors } & \multicolumn{9}{|c|}{ Variables } \\
\hline & $\begin{array}{l}\text { SW } \\
(\mathrm{kg})\end{array}$ & $\begin{array}{l}C Y \\
(\%)\end{array}$ & $\begin{array}{l}\mathrm{FE} \\
(\%)\end{array}$ & $\begin{array}{l}\mathrm{FT} \\
(\%)\end{array}$ & $\begin{array}{l}\mathrm{AF} \\
(\%)\end{array}$ & $\begin{array}{l}\mathrm{LV} \\
(\mathrm{g})\end{array}$ & $\begin{array}{l}\mathrm{HT} \\
(\mathrm{g})\end{array}$ & $\begin{array}{l}\mathrm{GZ} \\
(\mathrm{g})\end{array}$ & $\begin{array}{l}\text { PV } \\
(\mathrm{g})\end{array}$ \\
\hline \multicolumn{10}{|l|}{ Nut. Plans } \\
\hline Nut. Plan 1 & $2.58^{a}$ & 70.28 & 11.59 & $2.58^{a}$ & 0.93 & 43.37 & 20.62 & $66.25^{\mathrm{ab}}$ & 9.87 \\
\hline Nut. Plan 2 & $2.42^{\mathrm{ab}}$ & 69.30 & 9.02 & $2.42^{\mathrm{ab}}$ & 1.11 & 40.00 & 17.37 & $72.12^{\mathrm{a}}$ & 10.50 \\
\hline Nut. Plan 3 & $2.52^{\mathrm{a}}$ & 70.75 & 10.53 & $2.52^{\mathrm{a}}$ & 0.97 & 39.25 & 19.25 & $58.87^{a b}$ & 13.50 \\
\hline Nut. Plan 4 & $2.36^{\mathrm{ab}}$ & 65.52 & 26.88 & $2.00^{\mathrm{b}}$ & 0.88 & 39.50 & 17.00 & $58.12^{\mathrm{ab}}$ & 8.75 \\
\hline Nut. Plan 5 & $2.31^{\mathrm{ab}}$ & 71.84 & 9.33 & $2.36^{\mathrm{ab}}$ & 0.72 & 39.00 & 21.00 & $53.62^{\mathrm{bc}}$ & 11.25 \\
\hline $\begin{array}{l}\text { Nut. Plan } 6 \\
\text { Sexes }\end{array}$ & $2.00^{\mathrm{b}}$ & 75.25 & 20.10 & $2.31^{\mathrm{ab}}$ & 0.83 & 34.50 & 16.87 & $48.25^{c}$ & 10.75 \\
\hline Male & $3.07^{a}$ & $71.90^{\mathrm{a}}$ & $17.61^{a}$ & $3.03^{a}$ & $0.77^{b}$ & $47.25^{a}$ & $22.83^{a}$ & $68.66^{a}$ & $12.41^{\mathrm{a}}$ \\
\hline Female & $1.67^{b}$ & $69.07^{b}$ & $11.54^{\mathrm{b}}$ & $1.68^{\mathrm{b}}$ & $1.05^{\mathrm{a}}$ & $31.29^{b}$ & $14.54^{b}$ & $50.41^{b}$ & $9.12^{b}$ \\
\hline Effect & \multicolumn{9}{|c|}{$p$ Value } \\
\hline Nut. Plans & $0.02^{*}$ & $0.80^{\text {ns }}$ & $0.58^{\text {ns }}$ & $0.01^{* *}$ & $0.47^{\text {ns }}$ & $0.79^{\text {ns }}$ & $0.40^{\text {ns }}$ & $0.01^{* *}$ & $0.12^{\text {ns }}$ \\
\hline Sexes & $0.01^{* *}$ & $0.05^{*}$ & $0.05^{*}$ & $0.01^{* *}$ & $0.02^{*}$ & $0.01^{* *}$ & $0.01^{* *}$ & $0.01^{* *}$ & $0.01^{* *}$ \\
\hline Interation & $0.23^{\text {ns }}$ & $0.25^{\text {ns }}$ & $0.30^{\text {ns }}$ & $0.33^{\text {ns }}$ & $0.35^{\text {ns }}$ & $0.45^{\text {ns }}$ & $0.28^{\text {ns }}$ & $0.32^{\mathrm{ns}}$ & $0.34^{\text {ns }}$ \\
\hline CV (\%) & 12.53 & 18.76 & 16.27 & 12.53 & 4.32 & 19.71 & 17.53 & 17.93 & 13.80 \\
\hline
\end{tabular}

$\mathrm{CV}=$ Coefficient of variation; "Means followed by lowercase letters in column differ in $5 \%$ by Tukey test $(p<0.05)$; * Means followed by lowercase letters in column differ in $1 \%$ by Tukey test $(p<0.01)$; ns = non-significant.

Even though the level of available phosphorus in the diets influencing the development of carcass, this did not represent influence on the \% of distribution of commercial cuts in carcass. However, birds fed nutritional plans 3 presented better results of $\%$ of thigh.

Male muscovy ducks presented more of $50 \%$ of carcass constituted by breast and back. Even males presented higher carcass, females presented better distribution of commercial cuts than males, with great distribution of cuts in its carcass. There was not interaction ( $p>0.05$ ) between factors.

Results of $\mathrm{pH}$ and physical measurements are present in table 6 . Differences $(p<0.05)$ were observed among nutritional plans for length, width and height of breast, and $\mathrm{pH}$ of leg. Differences $(p<0.05)$ were observed among sexes for length, width and height of breast and leg.

Birds fed nutritional plan 3 presented better breast and leg development. Nutritional plans with lower levels of available phosphorus presented worse development and lower $\mathrm{pH}$ values. There was not interaction ( $p>0.05$ ) between factors.

Male muscovy ducks presented more size of breast and leg, with great difference in the development of carcass among sexes. There was not interaction ( $p>0.05$ ) between factors.

Results of mineral composition and resistance of bones are present in table 7 . Differences $(p<0.05)$ were observed in all variables of mineral composition. Birds 
Table 5 - Commercial cuts of muscovy ducks in housing fed nutritional plans with different levels of available phosphorus.

\begin{tabular}{|c|c|c|c|c|c|c|}
\hline \multirow[b]{2}{*}{ Factors } & \multicolumn{6}{|c|}{ Variables } \\
\hline & $\operatorname{Neck}(\%)$ & Breast (\%) & Wing (\%) & Thigh (\%) & Drumstick (\%) & Back (\%) \\
\hline \multicolumn{7}{|l|}{ Nut. Plans } \\
\hline Nut. Plan 1 & 10.61 & 26.48 & 17.57 & $9.95^{b}$ & 11.11 & 24.68 \\
\hline Nut. Plan 2 & 10.62 & 25.55 & 17.56 & $11.21^{\mathrm{ab}}$ & 10.92 & 24.14 \\
\hline Nut. Plan 3 & 10.59 & 25.19 & 17.10 & $12.32^{\mathrm{a}}$ & 9.28 & 25.52 \\
\hline Nut. Plan 4 & 10.62 & 25.98 & 17.42 & $12.99^{a}$ & 9.26 & 23.73 \\
\hline Nut. Plan 5 & 10.04 & 26.04 & 17.87 & $12.09^{a b}$ & 9.50 & 24.06 \\
\hline Nut. Plan 6 & 10.26 & 24.94 & 16.68 & $12.19^{\mathrm{ab}}$ & 9.56 & 26.37 \\
\hline \multicolumn{7}{|l|}{ Sexes } \\
\hline Male & $10.88^{b}$ & $26.60^{a}$ & $16.27^{b}$ & $10.65^{b}$ & $8.86^{b}$ & $26.74^{a}$ \\
\hline Female & $11.13^{\mathrm{a}}$ & $24.39^{b}$ & $17.46^{a}$ & $12.74^{\mathrm{a}}$ & $10.12^{\mathrm{a}}$ & $24.16^{b}$ \\
\hline Effect & \multicolumn{6}{|c|}{$p$ Value } \\
\hline Nut. Plans & $0.91^{\mathrm{ns}}$ & $0.71^{\mathrm{ns}}$ & $0.72^{\text {ns }}$ & $0.01^{\star *}$ & $0.11^{\mathrm{ns}}$ & $0.47^{\text {ns }}$ \\
\hline Sexes & $0.05^{*}$ & $0.02^{*}$ & $0.05^{*}$ & $0.04^{*}$ & $0.01^{* *}$ & $0.01^{* \star}$ \\
\hline Interation & $0.25^{\text {ns }}$ & $0.26^{\text {ns }}$ & $0.59^{\text {ns }}$ & $0.65^{\text {ns }}$ & $0.35^{\text {ns }}$ & $0.46^{\mathrm{ns}}$ \\
\hline CV $(\%)$ & 20.35 & 11.55 & 8.99 & 13.90 & 16.53 & 15.13 \\
\hline
\end{tabular}

$\mathrm{CV}=$ Coefficient of variation; " Means followed by lowercase letters in column differ in $5 \%$ by Tukey test $(p<0.05)$; ** Means followed by lowercase letters in column differ in $1 \%$ by Tukey test $(p<0.01) ; n s=$ non-significant.

Table 6 - Physical measurements of breast and leg (thigh + drumstick) of muscovy ducks in housing fed nutritional plans with different levels of available phosphorus.

\begin{tabular}{|c|c|c|c|c|c|c|c|c|}
\hline \multirow{3}{*}{ Factors } & \multicolumn{8}{|c|}{ Variables } \\
\hline & \multicolumn{4}{|c|}{ Breast } & \multicolumn{4}{|c|}{ Leg (thigh + drumstick) } \\
\hline & Length $(\mathrm{cm})$ & Width $(\mathrm{cm})$ & Height $(\mathrm{cm})$ & $\mathrm{pH}$ & Lenght $(\mathrm{cm})$ & Width $(\mathrm{cm})$ & Height $(\mathrm{cm})$ & $\mathrm{pH}$ \\
\hline \multicolumn{9}{|l|}{ Nut. Plans } \\
\hline Nut. Plan 1 & $22.12^{\mathrm{a}}$ & $14.78^{\mathrm{a}}$ & $5.50^{\mathrm{a}}$ & 6.38 & 17.00 & 9.62 & 2.78 & $6.35^{\mathrm{a}}$ \\
\hline Nut. Plan 2 & $21.81^{\mathrm{ab}}$ & $14.75^{\mathrm{a}}$ & $5.06^{\mathrm{ab}}$ & 6.12 & 17.31 & 9.93 & 3.06 & $6.37^{a}$ \\
\hline Nut. Plan 3 & $21.75^{\mathrm{ab}}$ & $14.68^{\mathrm{a}}$ & $4.68^{\mathrm{ab}}$ & 6.12 & 17.12 & 10.00 & 2.62 & $6.29^{\mathrm{ab}}$ \\
\hline Nut. Plan 4 & $21.50^{\mathrm{ab}}$ & $11.81^{\mathrm{ab}}$ & $4.93^{\mathrm{ab}}$ & 6.28 & 17.18 & 9.31 & 2.62 & $6.27^{\mathrm{ab}}$ \\
\hline Nut. Plan 5 & $19.62^{a b}$ & $11.16^{b}$ & $4.06^{\mathrm{ab}}$ & 6.21 & 15.68 & 9.00 & 3.06 & $6.10^{\mathrm{b}}$ \\
\hline Nut. Plan 6 & $19.00^{b}$ & $11.05^{b}$ & $3.87^{\mathrm{b}}$ & 6.16 & 15.37 & 8.75 & 2.68 & $6.08^{b}$ \\
\hline \multicolumn{9}{|l|}{ Sexes } \\
\hline Male & $23.39^{a}$ & $14.35^{\mathrm{a}}$ & $5.02^{\mathrm{a}}$ & 6.23 & $18.29^{a}$ & $10.25^{\mathrm{a}}$ & $3.09^{a}$ & 6.25 \\
\hline Female & $18.54^{b}$ & $11.70^{\mathrm{b}}$ & $4.35^{b}$ & 6.19 & $14.93^{b}$ & $8.62^{b}$ & $2.52^{\mathrm{b}}$ & 6.24 \\
\hline Effect & \multicolumn{8}{|c|}{$p$ Value } \\
\hline Nut. Plans & $0.01^{* *}$ & $0.01^{* *}$ & $0.02^{*}$ & $0.06^{\text {ns }}$ & $0.02^{\text {ns }}$ & $0.72^{\text {ns }}$ & $0.50^{\text {ns }}$ & $0.01^{* \star}$ \\
\hline Sexes & $0.01^{* *}$ & $0.01^{* *}$ & $0.02^{*}$ & $0.52^{\text {ns }}$ & $0.01^{* *}$ & $0.01^{* *}$ & $0.01^{* *}$ & $0.74^{\text {ns }}$ \\
\hline Interation & $0.12^{\text {ns }}$ & $0.28^{\text {ns }}$ & $0.06^{\text {ns }}$ & $0.15^{\text {ns }}$ & $0.14^{\text {ns }}$ & $0.25^{\mathrm{ns}}$ & $0.07^{\text {ns }}$ & $0.19^{\text {ns }}$ \\
\hline CV (\%) & 9.39 & 15.87 & 21.76 & 2.88 & 10.49 & 20.19 & 22.05 & 2.80 \\
\hline
\end{tabular}

$\mathrm{CV}=$ Coefficient of variation; * Means followed by lowercase letters in column differ in $5 \%$ by Tukey test $(p<0.05)$; ${ }^{* *}$ Means followed by lowercase letters in column differ in $1 \%$ by Tukey test $(p<0.01)$; ns = non-significant.

Table 7 - Mineral composition (ashes, calcium and phosphorus) and resistance of bones of muscovy ducks in housing fed nutritional plans with different levels of available phosphorus.

\begin{tabular}{lccccc}
\hline & \multicolumn{3}{c}{ Variables } \\
\cline { 2 - 5 } Nutritional plans & Ashes (\%) & Ca (\%) & $p(\%)$ & Ca:P & Break resistance (N) \\
\hline Nut. Plan 1 & 51.95 & $15.60^{\mathrm{ab}}$ & $9.30^{\mathrm{ab}}$ & $1.68^{\mathrm{ab}}$ & 430.55 \\
Nut. Plan 2 & 53.21 & $15.70^{\mathrm{a}}$ & $8.60^{\mathrm{b}}$ & $1.83^{\mathrm{a}}$ & 413.81 \\
Nut. Plan 3 & 51.36 & $14.47^{\mathrm{b}}$ & $8.58^{\mathrm{b}}$ & $1.69^{\mathrm{ab}}$ & 444.69 \\
Nut. Plan 4 & 49.74 & $14.36^{\mathrm{b}}$ & $9.65^{\mathrm{a}}$ & $1.49^{\mathrm{bc}}$ & 387.77 \\
Nut. Plan 5 & 51.85 & $11.31^{\mathrm{c}}$ & $8.34^{\mathrm{b}}$ & $1.36^{\mathrm{c}}$ & 423.10 \\
Nut. Plan 6 & 50.01 & $14.18^{\mathrm{b}}$ & $9.18^{\mathrm{ab}}$ & $1.54^{\mathrm{bc}}$ & 329.51 \\
\hline$p$ Value & $0.72^{\mathrm{ns}}$ & $0.01^{\star}$ & $0.01^{*}$ & $0.01^{*}$ & $0.10^{\text {ns }}$ \\
\hline CV (\%) & 4.76 & 1.03 & 1.10 & 1.25 & 8.23 \\
\hline
\end{tabular}

$\mathrm{CV}=$ Coefficient of variation; " Means followed by lowercase letters in column differ in $1 \%$ by Tukey test $(p<0.01)$; ns = non-significant. 
fed nutritional plans with higher levels of available phosphorus presented larger mineral deposition on bone, without affecting the break resistance. Nutritional plans with lower levels of available phosphorus presented more fragile bones.

\section{DISCUSSION}

In ourstudy, even without differenceson performance results, muscovy ducks presented available phosphorus requirements above recommendations for broilers at all phases. According to Feijó et al. (2016), higher mineral requirements for muscovy ducks are attributed to its greater carcass conformation and bone structure, larger than broilers.

Pinheiro (2009) observed better feed conversion of slow-growing broilers (both sexes) in free-range system fed diets with available phosphorus levels among 0.25 to $0.36 \%$ in the initial phase ( 1 to 28 days). Runho et al. (2001) studying other nutritional plans, observed better feed conversion of broilers in the initial phase (1 to 21 days) fed diets with available phosphorus levels among 0.15 to $0.45 \%$. Our results indicate a requirement near to these recommendations.

According Rostagno et al.(2005), Pinheiro (2009) and Rostagno et al.(2011), birds for meat production (broilers, ducks or muscovy ducks), present higher requirements of available phosphorus due to its larger and faster body development, with ideal levels according to the phase and profile of nutritional plan used.

These results reflected on carcass traits, where higher levels of available phosphorus presented a positive influence on muscovy ducks carcass development, similar results observed by Feijó et al. (2016) studding calcium levels for muscovy ducks, and obeying the 2:1 ratio between $\mathrm{Ca}$ and $\mathrm{P}$.

Macari et al. (2002) commented that calcium and phosphorus are associated elements. These are almost always combined ( 2 molecules of calcium for 1 molecule of phosphorus), and the deficiency of one in the diet limits the birds' performance (McDowell, 1992).

Nelson \& Peeler (1961), report that levels of phosphorus below or above the requirement difficult the birds' development, especially due to bone mineralization. Thus, Macari et al. (2002) and Feijó et al. (2016) affirm that the better balance among calcium and phosphorus requirements, and their metabolic relationship, provide better performance and development.
Higher available phosphorus levels were sufficient to meet the nutritional requirements, presented better results of breast and leg and with males presented good development of the main commercial cuts. There was also a greater deposition of minerals in the tibia from higher available phosphorus levels.

Runho et al. (2001) observed an increase in bone minerals of broilers (males and females) at 1 to 21 days from available phosphorus levels among 0.15 to $0.45 \%$, below the requirements obtained for muscovy ducks in the same period.

Another important question is the most requirement of available phosphorus in the initial phase for broilers and muscovy ducks, that according to Macari et al. (2002) and Sousa et al. (2015), is due to the faster growth of bone tissue than other tissues, with Ca and $\mathrm{P}$ deposition more necessary at this stage than others. However, there was no influence of available phosphorus levels on mineral composition and bone resistance of muscovy ducks.

Our results also presented a great difference between male and female carcass. Males presented larger carcass than females. According Gois et al. (2012), this could be attributed to better feed efficiency of males than females in the same period, presenting a significant difference in weight gain, slaughter weight, $\%$ of feathers, $\%$ of feet and edible viscera.

Yakubu (2010), Gois et al. (2012), and Almeida (2016) comment that a natural sexual dimorphism for muscovy ducks exists, with mean weight of 3.80 $\mathrm{kg}$ for males and $2.22 \mathrm{~kg}$ for females. But, Drumond et al. (2013) and Almeida (2016) affirm that females present a precocity growth, reaching the adult weight faster, better distribution of commercial cuts and faster ideal carcass fat deposition (Vieira,1999), even though having a lower final weight.

Stringhini et al. (2003) affirms that females have great carcass fat deposition due its present adipocytes with larger size than the males, which indirectly cause a lower feed efficiency (Mignon-Grasteau et al., 2000).

The sex is one of the factors that most affects the breast yield of birds (Rosa et al., 2006). Studies with broilers presented that males have higher breast than females, mainly due the reduction of meat deposition in breast at 42 days, when it has reached the maturity, which does not occur in females (Mendes et al., 2003). Our results presented that muscovy ducks males had a higher breast yield (26.60\%) than females (24.39\%), as well other carcass traits.

All these informations are important to elaborate strategies for Muscovy ducks production in industrial 
scale, aiming to meet great consumer markets, such as China, Japan, France, Germany and others countries (Cruz et al., 2013; Minas State Journal, 2015).

\section{CONCLUSIONS}

The present study indicates that nutritional plan 2 (initial $=0.60 \%$; growth $=0.55 \%$ and termination $=$ $0.50 \%$ ) presented ideal nutritional requirements of available phosphorus for muscovy ducks in housing, with better carcass development and mineral deposition on bones.

\section{REFERENCES}

ABPA - Associação Brasileira de Proteína Animal. Relatório Anual 2018. São Paulo: ABPA; 2018.

Almeida ECJ, Santos MRA, Farias Filho SRV, Hora FF, Oliveira EB, Pereira $A H R$, et al. Variabilidade fenotípica de características de carcaça do pato nativo comparada com linhagem comercial. Anais da $24^{\text {a }}$ Semana de Zootecnia da UFRPE; 2016; Recife. Pernambuco. Brasil: UFRPE; 2016.

AOAC - Association of Official Analytical Chemists. Official methods of analysis of the Association of Official Analytical Chemists. 16 ${ }^{\text {th }}$ ed. Washington: AOAC; 1999

Cruz FGG, Maquiné LC, Chagas EO, Melo JBS, Chaves FAL. Desempenho de patos (Cairinamoschata) em confinamento submetidos a diferentes densidades de alojamento. Revista Acadêmica: Ciências Agrárias e Ambientais 2013;11(3):313-319.

Drumond ESC, Gonçalves FM, Veloso RC, Amaral JM, Balotin LV, Pires AV, et al. Curvas de crescimento para codornas de corte. Ciência Rural 2013:43(10):1872-1877

Dunbar MR, Gregg MA, Crawford JA, Giordano MR, Tornquist SJ. Normal hematologic and biochemical values for prelaying greater sage grouse (Centrocercus urophasianus) and their influence on chick survival. Journal of Zoo and Wildlife Medicine 2005;36(3):422-429.

Feijó JC, Cruz FGG, Rufino JPF, Melo RD, Melo LD, Costa APGC, et al. Planos nutricionais com diferentes níveis de cálcio sobre o desempenho e rendimentos de carcaça de patos (Cairina moschata) em confinamento. Revista Científica de Avicultura e Suinocultura 2016;2(1):011-020.

Gois FD, Almeida ECJ, Farias Filho RV, Silva Filha OL. Estudo preliminar sobre o dimorfismo sexual do pato cinza do catolé (Cairinamoschata). Actas Ibero Americanas de Conservacion Animal 2012;2:95-98.

Gomide LAM, Alencar N, Macedo IA. Processamento de frango (corte, recorte e desossa). $2^{\text {nd }}$ ed. Brasília: LK Editora; 2012

Industrial Poultry. Industrialização de patos e marrecos [cited 2017 Out 15]. 2005. Available from: goo.gl/WcQ4jj.

Macari M, Furlan RL, Gonzáles E. Fisiologia aviária aplicada a frangos de corte. Jaboticabal: Ed. Funep; 2002. v.2.

Mariante AS, Albuquerque MSM, Ramos AF. Criopreservação de recursos genéticos animais brasileiros. Revista Brasileira de Reprodução Animal 2011;35(2):64-68.

McDowell LR. Minerals in animal and human nutrition. New York: Academic Press; 1992.

Mendes AA, Moreira J, Garcia RG. Qualidade da carne de peito de frango de corte. Revista Nacional da Qualidade da Carne 2003;28(317).
Mignon-Grasteau S, Piles M, Varona L, de Rochambeau H, Poivey JP, Blasco $A$, et al. Genetic analysis of growth curve parameters for male and female chickens resulting from selection on shape of growth curve. Journal of Animal Science 2000;78(10):2515-2524

Minas State Journal. Carne de pato está em plena valorização, mas faltam criadores em Minas [cited 2017 Out 15]. 2015. Available from: https:// goo.gl/64i6E1.

Nelson TS, Peeler HJ. The availability of phosphorus from single and combined phosphates to chicks. Poultry Science 1961;40:1321-1328.

Pinheiro SRF. Níveis de fósforo, de cálcio e de cloreto de sódio para aves de linhagem de crescimento lento criadas em sistema semiconfinado [tese]. Jaboticabal (SP): Faculdade de Ciências Agrárias e Veterinárias,Universidade Estadual Paulistal; 2009. 104p.

Pinheiro SFR, Sakomura NK, Nascimento DCN, Dourado LRB, Fernandes JBK, Thomaz MC. Níveis nutricionais de fósforo disponível para aves de corte ISA Label criadas em semiconfinamento. Brazilian Journal of Animal Science 2011;40(2):361-369

Rosa FC, Bressan MC, Bertechini AG, Fassani EJ, Oliveira e Vieira J, Faria $\mathrm{PB}$, et al. Efeito de métodos de cocção sobre a composição química e colesterol em peito e coxa de frangos de corte. Ciência e Agrotecnologia 2006;30(4):707-714.

Rostagno HS, Albino LFT,Donzele JL, Gomes PC, Oliveira RF, Lopes DC, et al. Tabelas brasileiras para aves e suínos: composição de alimentos e exigências nutricionais. $2^{\text {nd }}$ ed. Viçosa: UFV; 2005.

Rostagno HS, Albino LFT, Donzele JL, Gomes PC, Oliveira RF, Lopes DC, et al. Tabelas brasileiras para aves e suinos: composição dos alimentos e requerimentos nutricionais. $3^{\text {rd }}$ ed. Viçosa: UFV; 2011.

Rufino JPF, Cruz FGG, Melo LD, Soares VM,Curcio UA, Damasceno JL, et al. Quality and sensory evaluation of meat of ducks (Cairina moschata) in confinement under different nutritional plans and housing densities. International Journal of Poultry Science 2015;14(1):44-48.

Runho RC, Gomes PC, Rostagno HS, Albino LFT, Lopes PS, Pozza PC. Exigência de fósforo disponível para frangos de corte machos e fêmeas de 1 a 21 dias de idade. Brazilian Journal of Animal Science 2001;30(1):187-196.

Santos MSV, Vieira SS, Tavares FB, Andrade PA, Manno MC, Costa HS, et al. Desempenho, carcaça e cortes de frangos caipira francês barré (Gris Barre Cou Plumé). Archivos de Zootecnia 2012;61(234):287-295.

Sousa JPL, Albino LFT, Vaz RGMV, Rodrigues KF, Da Silva GF, Renno LN, et al. The effect of dietary phytase on broiler performance and digestive, bone, and blood biochemistry characteristics. Brazilian Journal of Poultry Science 2015;17(1):69-76.

Stringhini JH, Laboissiére M, Muramatsu K, Leandro NSM, Café MB. Avaliação do desempenho e rendimento de carcaça de quatro linhagens de frangos de corte criadas em Goiás. Brazilian Journal of Animal Science 2003;32(1):183-190

Vieira SL. Considerações sobre as características de qualidade de carne de frango e fatores que podem afetá-la. Anais da 36 Reunião Anual da Sociedade Brasileira de Zootecnia; 1999; Porto Alegre(RS): SBZ; 1999.

Wawro K, Wilkiewicz-Wawro E, Kleczek K, Brzozowski W. Slaughter value and meat quality of muscovy ducks, pekin ducks and their crossbreds, and evaluation of the heterosis effect. Archiv Tierzucht 2004;47:287299

Yakubu A. Characterization of the local muscovy duck genetic resource of Nigeria and its potential for egg and meat production. World'sPoultry Science Journal 2013;69(4):931-938. 
